\title{
Communication with general practitioners: a survey of spinal cord injury physicians' perspectives
}

\author{
Michael Stillman ${ }^{1,2} \cdot$ Kristin Gustafson $^{2} \cdot$ Guy W. Fried $^{3} \cdot$ Karen Fried $^{4} \cdot$ Steve R. Williams ${ }^{2}$
}

Received: 1 March 2019 / Revised: 17 April 2019 / Accepted: 20 April 2019

(c) International Spinal Cord Society 2019

\begin{abstract}
Study design An online questionnaire.

Objectives To gauge spinal cord injury (SCI) specialists' assessment of their communications with general practitioners (GPs). To determine whether economic or health-care system-related factors enhance or inhibit such communication.

Setting A collaboration of co-authors from a health-care system.

Methods An online survey interrogating a number of aspects of communication between SCI specialists and GPs was developed, distributed, and made available for 4 months. Responses were analyzed for the entire cohort then according to descriptions of participants' home nations' economies and the type of health-care delivery systems in which they work.

Results A total of 88 responses were submitted. The majority (64\%) were from nations with developed economies, a plurality $(47.1 \%)$ were from countries that offer universal health coverage, and half used a combination of paper and electronic health records. A majority of respondents $(61.8 \%)$ reported routinely communicating with their patients' GPs, but most $(53.4 \%)$ rated those communications as only "fair". The most commonly listed barriers to communication with GPs were lack of time (46.3\%) and a perceived lack of receptivity by GPs (26.9\%). Nearly all respondents (91.6\%) believed that the care they provide would be enhanced by improved communication with GPs. Participants who used electronic means of communication were more likely to communicate with GPs and to describe those interactions as "positive".

Conclusions Although there are a number of barriers to communication between SCI specialists and GPs, most SCI specialists are eager for such inter-physician communication and believe it would enhance their care they deliver.
\end{abstract}

\section{Background}

Many people living with spinal cord injury (SCI) receive care from both SCI specialists and general practitioners (GPs). In Stillman et al.'s survey of 108 individuals with

Michael Stillman

michael.stillman@jefferson.edu

1 Internal Medicine and Rehabilitation Medicine, Sidney Kimmel Medical College of Thomas Jefferson University, 1100 Walnut Street, Suite 601, Philadelphia, PA 19107, USA

2 Department of Rehabilitation Medicine, Sidney Kimmel Medical College of Thomas Jefferson University, Philadelphia, PA, USA

3 Senior Vice President for Medical Affairs and Chief Medical Office, Magee Rehabilitation Hospital, Department of Rehabilitation Medicine, Sidney Kimmel Medical College of Thomas Jefferson University, Philadelphia, PA, USA

4 Magee Outpatient Physician Practice Clinic, Magee Rehabilitation Hospital, Philadelphia, PA, USA
SCI, a plurality of respondents had made between 3 and 5 visits to both an SCI specialist and a GP within the past year [1]. In Donnelly et al.'s study of how people with SCI in the United States, Canada, and the United Kingdom utilize physician services [2], 93\% of respondents had a GP, $63 \%$ had an SCI specialist, and 56\% had both. Of note, there was substantial overlap between the medical concerns participants would bring to their SCI specialists and to their GPs.

Although people with SCI may turn to their GPs for care, many generalists may be inadequately prepared to assist them. First, GPs' offices are frequently inaccessible to people with SCI. In Stillman et al. [1], 91\% of subjects with SCI reported having faced an accessibility barrier while seeking primary care and the majority were encountered inside examination rooms. In the study by Donnelly et al. [2], only $56 \%$ of respondents were able to use all the equipment in their GP's office. Second, many GPs lack specific training in the care of people with SCI and other complex disabilities. In Premo et al.'s [3] survey of physicians in California, only $22 \%$ reported having received 
education pertaining to the care of such individuals, whereas in Holder et al.'s [4] study of medical students and attending physicians, the majority felt unprepared to treat their patients with disabilities. This oversight in medical training has real clinical consequences. Among Stillman et al.'s respondents, only $36 \%$ believed that their GPs understood their disability-specific concerns "well or very well" [1].

Given persistent challenges surrounding GPs' office accessibility and medical training, it is not surprising that some people with SCI turn to SCI specialists for attention to their general and preventative health concerns. In Johnston et al.'s [5] survey of men with SCI, 59\% considered their physiatrist to be their "main doctor type", and in Donnelly et al. [2], 54\% of respondents had approached their SCI specialist for an annual physical examination. Despite this phenomenon, however, some SCI specialists feel uncomfortable providing primary care. In the only study to examine physiatrists' attitudes toward serving as GPs [6], only 53\% believed that they ought to be GPs for people with disabilities or that they had the requisite knowledge to do so.

With GPs being sub-optimally prepared to care for people with SCI and with SCI specialists being reluctant to serve as GPs, communication between the two seems essential. However, this specific form of inter-professional collaboration has remained essentially uninvestigated. In this study, we distributed a survey assessing SCI specialists' interest in and assessment of their communication with GPs, and whether these communications are influenced by the economies and the health-care systems in which they work. Although our findings are preliminary, we hope they will serve as the basis for further study into how to offer more comprehensive and seamless care to individuals living with SCI.

\section{Methods}

Our online survey was developed and distributed (from November of 2018 through February of 2019) to an international cohort of physicians who care for individuals with SCI. The survey was developed by all five authors (four SCI specialists and one GP) based on their expertise in the field then administered electronically (SurveyMonkey Inc, San Mateo, CA, www.surveymonkey.com) to their colleagues in the field of SCI Medicine and to members of the International Spinal Cord Society (ISCOS) via its online newsletter. No identifying information was collected, and consent was implied by participants' completing the survey. No ethics approvals were obtained or considered necessary for this project.
Descriptive analyses were performed using Chi Square tests. Responses were analyzed for the entire subject pool, then separated according to participants' descriptions of their home nations' economies ("Developed", "Transitional", or "Developing"), health-care systems ("Universal/ government funded", "Tier-based system, basic universal healthcare with available private coverage", or "No universal health care; system of private/public coverage"), and methods of medical record keeping (paper records, electronic records, or a mix of the two). As fewer responses were submitted from participants living in countries with developing $(n=16)$ or transitional $(n=6)$ economies than from participants working in developed economies $(n=$ $64)$, the first two were combined into the category "Developing Nation". To bring clarity to other analyses, certain groups and answers were combined, and this is noted in the results.

\section{Results}

Eighty-eight physicians responded to the survey. The majority $(64 \%)$ were from nations with developed economies, $47.1 \%$ were from countries with universal health coverage $29.9 \%$ from tiered systems with basic universal coverage and $23.0 \%$ from systems without universal coverage), and 50\% used a mix of paper and electronic health records (EHR) in their practices $(41.9 \%$ used exclusively electronic records and $8.1 \%$ used paper only). Just over $71 \%$ reported that their patients with SCI also see GPs (20.5\% replied "no" and $8 \%$ that they did not know), a plurality $(47.6 \%)$ felt comfortable serving as a GP for their patients with SCI (33.3\% replied "no" and 19.1\% replied "not sure"), and whereas $61.8 \%$ routinely communicated with their patients' GPs, the majority of them $(53.4 \%)$ rated those communications as only "fair" (11.1\% "excellent", $24.4 \%$ "good", and $11.1 \%$ "poor"). Nearly all respondents $(91.6 \%)$ felt that the care they provided to their patients with SCI would be enhanced by improved communication with GPs.

Among the participants who routinely communicated with their patients' GPs $(n=47), 40.0 \%$ reported primarily corresponding by mail or fax and $28.9 \%$ by phone $(15.6 \%$ through EHR, $11.1 \%$ in person, and $4.4 \%$ through other secure messaging systems). When these same respondents were asked how their patients' GPs communicate with them, $35.6 \%$ replied "by phone", $20 \%$ "by mail or fax", and $17.8 \%$ that their GP colleagues do not communicate with them. When asked to identify the strongest barrier to improved communication with GPs, $46.3 \%$ selected "lack of time", $16.4 \%$ the "administrative burden", and $10.5 \%$ the difficulty of accessing secure platforms. However, over one- 
quarter $(26.9 \%)$ felt that a perceived lack of receptivity by GPs was the primary obstacle to enhanced correspondence.

Participants' home nations' economies did not determine the type of health-care system in which they practiced. Respondents from developed nations were no more likely than those from developing nations to work in a system with universal coverage (51.6\% vs $34.8 \%$; NS). Neither participants' home nations' economic status nor the type of health-care system in which they worked influenced whether their patients also saw a GP, their comfort with serving as a GP for individuals with SCI, whether they routinely communicated with GPs, or their means of communicating with GPs.

In grouping certain answers for analysis, several significant associations emerged. When we combined two possible responses to type of medical records used (paper only and mix of paper and electronic records), we found that respondents from developed nations were more likely than those from developing nations to exclusively use EHRs (50.8\% vs $17.4 \% ; p=0.005)$. When we combined responses to satisfaction with communication with GPs into "positive" ("excellent" plus "good") and "negative" ("fair" plus "poor"), we found that participants using electronic means of communication were more likely than those using phone or fax to routinely communicate with GPs (100\% vs $68.9 \%$ ) and to rate those communications as positive (44.4\% vs $22.6 \%$; $p=0.003)$.

\section{Discussion}

To our knowledge, this is the first study to evaluate SCI specialists' perspectives on their communication with GPs and to begin to determine which factors promote or hinder those communications. Although our sample size was small, limiting our analyses and ability to draw conclusions, several findings are notable.

First, our results are in line with those of prior studies showing that most individuals with SCI see both an SCI specialist and a GP. In the study by Donnelly et al. [2], respondents from countries with universal health coverage were more likely to have a GP than were those from the United States (99\% in the United Kingdom, 97\% in Canada, and $76 \%$ in the United States). In this study, neither the state of economic development nor the system of healthcare influenced whether individuals with SCI were co-managed by GPs. Our data provide a simple reiteration of the fact that many people with SCI knit together a complex network of care, and that like other people with chronic medical conditions, turn to a variety of physicians and health-care providers for assistance.

Second, our results show that SCI specialists have an unequivocal interest in improved communication with their patients' GPs, but that nearly $27 \%$ of them perceive that interest is not reciprocated. People living with acute and chronic SCI have a high risk of hospitalization [7, 8] and of several potentially life-threatening secondary effects of paralysis [9-11]. Although communication between SCI specialists and GPs has never been formally studied nor shown to improve clinical outcomes, it seems evident that it would. Future studies may investigate how to better engage GPs in the multi-specialty care of their patients with SCI and whether that engagement would result in a reduction in re-hospitalization and SCI-related complications.

Third, our findings suggest that clinical practices and health-care systems must continue to explore technologies that facilitate inter-physician communication. Many researchers have shown that adoption of EHRs shifts the amount of time traditionally spent in a variety of aspects of patient care [12-14], an early manuscript demonstrated that emergency room physicians were eager to use electronic modalities to communicate with community-based colleagues [15], and several authors have demonstrated that use of an EHR can improve the quality and efficiency of discharges from inpatient services and of communications between physicians, nurses, and pharmacists [16-18]. However, only one study to our knowledge has shown that adoption of electronic technologies-specifically, a computerized referral platform-enhances quality or frequency of inter-physician communications [19], and our data may support that notion. Future work ought to explore whether this association holds, and if it does, how to make these technologies more available and affordable to health-care providers working in less developed nations.

\section{Limitations}

This work has several limitations. First, our sample size was small, potentially obscuring findings that would emerge from a larger data set. Second, we only invited physicians to take our study, which excluded the insights and experiences of clinicians such as nurse practitioners and physician assistants who often provide front-line care to individuals with SCI. Future iterations of this study will certainly be more inclusive. Finally, participants from less-developed nations were under-represented in this study. Moving forward, we must focus our energies on improving representation of colleagues from a variety of nations. This being said, this study is the first to investigate SCI specialists' perspectives on their communications with GPs and to attempt to determine which factors facilitate and retard those communications. It may serve as stimulus for more-detailed work addressing these questions. 


\section{Compliance with ethical standards}

Conflict of interest The data sets generated and analyzed during the current study are available from the corresponding author on reasonable request. The authors declare that they have no conflict of interest.

Publisher's note: Springer Nature remains neutral with regard to jurisdictional claims in published maps and institutional affiliations.

\section{References}

1. Stillman MD, Frost KL, Smalley C, Bertocci G, Williams S. Health care utilization and barriers experienced by individuals with spinal cord injury. Arch Phys Med Rehabil. 2014;95:1114-26.

2. Donnelly C, McColl MA, Charlifue S, Glass C, O'Brien P, Savic $\mathrm{G}$, et al. Utilization, access and satisfaction with primary care among people with spinal cord injuries: a comparison of three countries. Spinal Cord. 2007;45:25-36.

3. Premo, B, Carrothers, L, and Larson-McNeal, M. Providing primary health care for people with physical disabilities: a survey of California physicians. Center for Disability Issues and the Health Profession, Western University of Health Sciences, Pomona, CA, USA, 2002.

4. Holder M, Waldman HB, Hood H. Preparing health professionals to provide care to individuals with disabilities. Int J Oral Sci. 2009;1:66-71.

5. Johnston MV, Diab ME, Chu BC, Kirshblum S. Preventive services and health behaviors among people with spinal cord injury. J Spinal Cord Med. 2005;28:43-54.

6. Francisco GE, Chae JC, DeLisa JA. Physiatry as a primary care specialty. Am J Phys Med Rehabil. 1995;74:186-92.

7. Cardenas DD, Hoffman JM, Kirshblum S, McKinley W. Etiology and incidence of rehospitalization after traumatic spinal cord injury: a multicenter analysis. Arch Phys Med Rehabil. 2004;85:1757-63.

8. DeJong G, Tian W, Hsieh CH, Junn C, Karam C, Ballard PH, et al. Rehospitalization in the first year of traumatic spinal cord injury after discharge from medical rehabilitation. Arch Phys Med Rehabil. 2013;94:S87-97.
9. New PW. Secondary conditions in a community sample of people with spinal cord damage. J Spinal Cord Med. 2016;39:665-70.

10. Charlifue SW, Weitzenkamp DA, Whiteneck GG. Longitudinal outcomes in spinal cord injury: aging, secondary conditions, and well-being. Arch Phys Med Rehabil. 1999;80:1429-34.

11. Anson CA, Shepherd C. Incidence of secondary complications in spinal cord injury. Int J Rehabil Res. 1996;19:55-66.

12. Sinsky C, Colligan L, Li L, Prgomet M, Reynolds S, Goeders L, et al. Allocation of physician time in ambulatory practice: a time and motion study in 4 specialties. Ann Intern Med. 2016;165:753-60.

13. Joukes E, Abu-Hanna A, Cornet R, de Keizer NF. Time spent on dedicated patient care and documentation tasks before and after the introduction of a structured and standardized electronic health record. Appl Clin Inform. 2018;9:46-53.

14. Tai-Seale M, Olson CW, Li J, Chan AS, Morikawa C, Durbin M, et al. Electronic health record logs indicate that physicians split time evenly between seeing patients and desktop medicine. Health Aff (Millwood). 2017;36:655-62.

15. Stiell AP, Forster AJ, Stiell IG, van Walraven C. Maintaining continuity of care: a look at the quality of communication between Ontario emergency departments and community physicians. CJEM. 2005;7:155-61.

16. Mehta RL, Baxendale B, Roth K, Caswell V, Le Jeune I, Hawkins $\mathrm{J}$, et al. Assessing the impact of the introduction of an electronic hospital discharge system on the completeness and timeliness of discharge communication: a before and after study. BMC Health Serv Res. 2017;17:624.

17. Driscoll M, Gurka D. Using the electronic medical record to enhance physician-nurse communication regarding patients' discharge status. Nurs Adm Q. 2015;39:E31-7.

18. Singer A, Duarte Fernandez R. The effect of electronic medical record system use on communication between pharmacists and prescribers. BMC Fam Pract. 2015;16:155.

19. Ghandi T, Keating N, Ditmore M, Kierman D, Johnson R, Burdick E, et al. Improving Referral Communication Using a Referral Tool Within an Electronic Medical Record. In: Henriksen K, Battles J, Keyes M, Grady M, eds. Advances in Patient Safety: New Directions and Alternative Approaches (Vol. 3: Performance and Tools). Rockville, MD: Agency for Healthcare Research and Quality; 2008. 